\title{
Sodalis Glossinidius and Wolbachia Infections in Wild Population of Glossina Morsitans Submorsitans Caught in the Area of Lake Iro in the South of Chad
}

\section{Djoukzoumka Signaboubo}

University of Dschang

Vincent Khan Payne

University of Dschang

Ibrahim Mahamat Alhaj Moussa

University of Bremen

Mahamat Hassane Mahamat

Institut de Recherche en Elevage pour le Développement

\section{Sartrien Tagueu Kanté}

University of Dschang

\section{Youssouf Mouliom Mfopit}

Institute of Agricultural Research for development

\section{Petra Berger}

University of Bremen

Soerge Kelm

University of Bremen

Gustave Simo ( $\nabla$ gsimoca@yahoo.fr)

University of Dschang

\section{Research Article}

Keywords: Glossina morsitans submorsitans, Sodalis glossinidius, Wolbachia, Lac-iro, Chad

Posted Date: April 15th, 2021

DOI: https://doi.org/10.21203/rs.3.rs-420061/v1

License: (c) (1) This work is licensed under a Creative Commons Attribution 4.0 International License.

Read Full License 


\section{Abstract}

Background: To improve vector control of tsetse transmitted trypanosomiases through a better understanging of the vectorial competence of tsetse flies, investigations have been undertaken on the bacterial fauna of different tsetse species. It is in this light that Wolbachia and Sodalis glossinidius were detected in wild populations of Glossina morsistans submorsistans caught in the area of lake Iro in the south of Chad with the aim of generating data that may help to understand the influence of these symbiotic microorganisms on the vectorial competence of G. m. submorsistans.

Methods: Tsetse flies were captured using biconical traps. DNA was extracted from tsetse body using the resin chelex. Sodalis glossinidius and Wolbachia were investigated by PCR using specific primers for pSG 2 and wsp genes. Comparisons between S. glossinidius and Wolbachia infections were performed according to sex and sampling periods and association studies between trypanosome infections and $S$. glossinidius or Wolbachia were performed.

Results: From 345 G. m. submorsitans analyzed, $9.0 \%$ and $14.5 \%$ were respectively infected with $S$. glossinidius and Wolbachia. Only $2.31 \%$ of all tsetse flies hosted the 2 bacteria. Of all trypanosomeinfected flies, $7.1 \%$ and $9.8 \%$ hosted respectively S. glossinidius and Wolbachia. No association was observed between Wolbachia and trypanosomes while significant association $(r=4.992 ; P=0.025)$ was found between $S$. glossinidius and trypanosome infections. Although significant association $(r=3.147 ; P$ $=0.043$ ) was observed between S. glossinidius and T. simiae, none was found with T. congolense or $T$. godfreyi.

Conclusion: This study revealed S. glossinidius and Wolbachia in wild population of G. m. submorsitans of lake Iro. It showed that few tsetse flies are co-infected by Wolbachia and S. glossinidius. The presence of $S$. glossinidius seems to favor trypanosome infections while Wolbachia has probably no effect. Decrypting the tripartite association requires to investigate the relationship between haplotypes or genotypes of Wolbachia and/or S. glossiniduis and trypanosome infections.

\section{Introduction}

Tsetse flies are biological vectors of African trypanosomes that cause human and animal African trypanosomiases respectively in humans and animals. Human African trypanosomiasis (HAT) is caused by two subspecies of trypanosomes: Trypanosoma brucei rhodesiense which is responsible of the acute form of HAT in eastern and southern Africa, and T. b. gambiense that causes the chronic form of HAT in western and central Africa [1]. About 65 million people are at risk of HAT and currently, the number of reported cases is, for the first time below 2000 new cases [2]. Control efforts undertaken in the last three decades have brought HAT under control and led to its inclusion into the WHO "roadmap for the interruption of transmission to human by 2030 [3].

Animal African trypanosomiases (AAT) are caused by several trypanosomes species and subspecies including for instance T. b. brucei, T. congolense, T. vivax and T. simiae. These diseases remain a 
constraint for animal production and agriculture development. In absence of vaccine for African trypanosomiases, the control strategies deployed against these infectious diseases rely mainly on the diagnosis and treatment of infected mammals and vector control. The development of drug-resistant trypanosomes could jeopardize the control measures aiming to eliminate the protozoan parasites through treatment of infected hosts. Moreover, the strategy relying on treatment of infected mammals cannot be applied on wild animals that could serve as reservoirs of different trypanosome species and subspecies. In such context, vector control remains a very important component for the management of African trypanosomiases [4]. Integrating vector control as key component of new control strategies is becoming crucial to achieve the complete interruption of HAT transmission and boost AAT control. In this light, several approaches including the setup of tsetse traps, screens or "tiny targets", the use of insecticide and the modification tsetse biotopes have been developed to fight tsetse flies [5]. Although the implementation of these approaches enabled to reduce tsetse populations in most settings, their sustainability remains challenging and some of these approaches have environmental impacts [6]. There is a need to develop innovative vector control methods that may not have such impacts. In recent decades, growing interests have been focused on factors enable to interfere with the vectorial competence of tsetse flies [7, 8]. It is in this light that interactions between trypanosomes, tsetse and symbiotic microorganisms have been investigated $[9,10]$. Three symbiotic microorganisms including Wigglesworthia glossinidia, Sodalis glossinidius and Wolbachia have been associated with tsetse.

Wigglesworthia glossinidia is an obligate primary symbiont on which tsetse depend for their vital physiological functions such as host fertility and immune maturation $[11,12,9]$. Sodalis glossinidius is a secondary and non-essential symbiont. Although its biological function remains unknown [13, 14], $S$. glossinidius has been suspected to play a role in the susceptibility of tsetse to trypanosome infections by favoring midgut establishment of trypanosomes through a complex biochemical mechanism $[13,15,11]$. Wolbachia are also non-essential symbionts found in a wide range of arthropods and nematodes $[16,17$, 18]. They are transmitted from mother to offspring and can protect their hosts against viral pathogens $[19,20]$. Abundant in both male and female germ-cells as well as somatic tissues, Wolbachia are able to induce cytoplasmic incompatibility which leads to embryonic death in tsetse flies [21]. With such ability, investigations on Wolbachia could improve vector control through the development of transgenic tsetse that have the ability of releasing specific molecules that can interfere with the establishment of trypanosomes.

In wild tsetse populations from several tsetse infested regions, $S$. glossinidius and Wolbachia have been reported in G. m. morsitans, G. m. centralis, G. f. fuscipes, G. austeni, G. pallidipes, G. p. palpalis, G. f. quanzensis and $G$. brevipalpis $[7,22,23,24,20 ; 25,26,27]$. Previous investigations on the tripartite association reported contrasting results in different ecosystems. A negative association has been reported between Wolbachia and trypanosomes in G. f. fuscipes, suggesting that the presence of Wolbachia could prevent trypanosome infections [27]. In tsetse of palpalis group, no association has been reported between Wolbachia and trypanosome infections [28]. For S. glossinidius, some authors reported a positive association between $S$. glossinidius and trypanosome infections [29] while others found that the presence of S. glossinidius does not seem to favor trypanosome infections in G. p. palpalis 
[25]. The tripartite association between tsetse, symbiotic microorganisms and trypanosome infections seems to vary according to tsetse species as well as ecological settings. Decrypting the role that each symbiotic microorganisms could play in the establishment and the development of trypanosomes in tsetse species of each ecological setting is importance for the understanding of vectorial competence of tsetse flies.

In the present study, Wolbachia and S. glossinidius were screened in wild populations of G. $m$. submorsistans caught in the area of Lake Iro in the south of Chad with the aim of generating data that may help to understand the influence of these symbiotic microorganisms on the vectorial competence of G. m. submorsistans.

\section{Results}

\section{Entomological survey}

During the three entomological surveys, 54 tsetse traps were set up and 617 tsetse flies were caught: 12 tsetse caught in November 2018, 323 in February 2019 and 282 in February 2020. The entomological data have been previously described in Djoukzoumka et al. (accepted in Parasites and Vectors). From the 617 caught flies, 359 non teneral flies were randomly selected and their identification revealed 345 (96.1\%) G. m. submorsitans, 11 (3.06\%) G. f. fuscipes and 3 (0.84\%) G. tachinoides. For the present study, only the $345 \mathrm{G}$. m. submorsitans were subjected to the molecular identification of $S$. glossinidius and Wolbachia. These 345 flies included 105 (30.4\%) females and 240 (69.6\%) males.

\section{Molecular identification of $S$. glossinidius}

A tsetse fly was considered as harboring $S$. glossinudius infections if, after electrophoresis on agarose gel (Fig. 2) and sequencing, a DNA fragment of 120 bp was obtained. In addition, the obtained sequences must also show at least $98 \%$ of similarity with those of $S$. glossinidius available in the data base.

Of the 345 G. m. submorsitans analysed, $31(9.0 \%)$ harboured S. glossinidius. The infection rates of $S$. glossinidius were $5.71 \%(6 / 105)$ in females and $10.41 \%(25 / 240)$ in males. Comparing the $S$. glossinidius infection rates between males and females, no significant difference $\left(X^{2}=1,975 ; P=0.16\right)$ was observed. According to sampling period, the infection rate of S. glossinidius was 18.8\% (2/11) in November 2018, 10.8\% (22/216) in February 2019 and 5.9\% (7/118) in February 2020. Between the sampling periods, no significant difference $\left(X^{2}=3.213 ; P=0.2201\right)$ was observed in the $S$. glossinidius infection rates (Table 1). 
Table 1

Infection rates of S. glossinidius and Wolbachia in G. m. submorsitans caught in the Lake Iro area according to the sampling period

\begin{tabular}{|lllll|}
\hline Sampling period & Number of tsetse flies analyzed & NTW (\%) & NTSG (\%) & NTWSG (\%) \\
\hline November 2018 & 11 & $2(18.8)$ & $2(18.8)$ & $0(0.0)$ \\
\hline February 2019 & 216 & $31(14.3)$ & $22(10.8)$ & $7(3.2)$ \\
\hline February 2020 & 118 & $17(14.0)$ & $7(5.9)$ & $1(0.8)$ \\
X2 & & 0.154 & 3.213 & 2.376 \\
P-value & & 0.926 & 0.201 & 0.305 \\
Total & $\mathbf{3 4 5}$ & $\mathbf{5 0 ( 1 4 . 5 )}$ & $\mathbf{3 1 ( 9 , 0 )}$ & $\mathbf{8 ( 2 . 3 )}$ \\
\hline
\end{tabular}

NTW: number of tsetse flies with Wolbachia infections; NTSG: number of tsetse flies found with Sodalis glossiniduis infections; NTWSG: number of tsetse flies with coinfections of Wolbachia and Sodalis glossinidius; (\%): infection rate

\section{Molecular identification of Wolbachia}

A sample was considered as having Wolbachia infection if after electrophoresis on agarose gel (Fig. 3) and sequencing, a DNA fragment of 513 bp was obtained. The obtained sequences must also have at least $98 \%$ of similarity with those of Wolbachia available in the database.

From the 345 G. m. submorsitans analyzed in the present study, 50 (14.5\%) were found with Wolbachia infections. The Wolbachia infection rates were $19.0 \%(20 / 105)$ and $12.5 \%(30 / 240)$ in females and males respectively. Comparing the Wolbachia infection rates, no significant difference $\left(X^{2}=2,527 ; P=\right.$ $0.11)$ was observed between males and females. The Wolbachia infection rate was $18.8 \%(2 / 11)$ in November 2018, 14.3\% (31/216) in February 2019 and 14.0\% (17/118) in February 2020. Between the sampling periods, no significant difference $\left(X^{2}=0.154 ; P=0.926\right)$ was observed in the Wolbachia infection rates (Table 1).

Of the 345 tsetse flies that were simultaneous analyzed for the presence of Wolbachia and $S$. glossinidius, 8 (2.31\%) were co-infected with these two symbiotic microorganisms.

\section{Trypanosome infections in G. m. submorsitans}

To evaluate the tripartite association between trypanosome infections, G. $m$. submorsitans and symbiotic microorganisms (S. glossinidius and Wolbachia), results of trypanosome infections in G. $m$. submorsitans were retrieved from the paper of Djoukzoumka et al. (accepted in Parasites and Vectors). From the 345 G. m. submorsitans analysed in this paper, $212(61.4 \%)$ were infected by different trypanosome species including 196 (56.7\%) single infections and $16(4.6 \%)$ mixed infections. The single infections were constituted of 68 (19.7\%) T. simiae, 55 (15.9\%) T. congolense savannah, 39 (11.3\%) T. vivax and 34 (9.8\%) T. goffreyi. Considering the fact that the life cycle of $T$. vivax occurred mainly in the 
mouthparts of tsetse flies, the single infections involving this parasite were excluded in the tripartite association. Thus, from 345 tsetse flies that were simultaneously analyzed for the presence of trypanosomes, Wolbachia and S. glossinidius, the 39 tsetse flies with single infections of T. vivax were excluded. The 16 flies with mixed infections involving T. vivax were considered in the tripartite analysis. For association studies, only 306 tsetse flies were considered.

\section{Coinfection of S. glossinidius and trypanosome}

Of the 306 tsetse flies that considered for association studies, coinfections between S. glossinidius and trypanosomes ( $\mathrm{S}+\mathrm{T}+)$ were found in 7.1\% (22/306) of them (Table 2). Nine (3.0\%; 9/306) tsetse flies (S + T-) without trypanosome infections were infected with $S$. Glossinidius. In the contrary, 155 (50.6\%; $155 / 306$ ) tsetse flies (S-T+) harbored trypanosome infections without $S$. glossinidius. Taking together all trypanosome infections, a significant association ( $r=4.992 ; P=0.025 ; 95 \% \mathrm{Cl}=[0.178-5.012])$ was observed between the presence of $S$. glossinidius and trypanosome infections in G. m. submorsitans. When each trypanosome species was considered for association studies, a significant association $(r=$ 3.147; $\mathrm{P}=0.043 ; 95 \% \mathrm{Cl}=[0.178-5.012])$ was observed between $S$. glossinidius and $T$. simiae while no significant association was observed for $T$. congolense $(r=1.066 ; P=0.901 ; 95 \% C l=[0.390-2.912 J)$ and T. godfreyi $(\mathrm{r}=0.608 ; P=0.509 ; 95 \% \mathrm{Cl}=[0.139-2.677)$ (Table 2$)$.

Table 2

Combined results of $S$. glossinidius and trypanosome infections

\begin{tabular}{|c|c|c|c|c|c|c|c|c|c|c|}
\hline $\begin{array}{l}\text { Trypanosome } \\
\text { infections }\end{array}$ & No & $\begin{array}{l}\mathrm{S}+ \\
\mathrm{T}-\end{array}$ & $\begin{array}{l}S+ \\
T+\end{array}$ & $\begin{array}{l}\mathrm{S}- \\
\mathrm{T}+\end{array}$ & S-T- & S+ & $T+$ & $\mathbf{r}$ & $\begin{array}{l}P \\
\text { value }\end{array}$ & $95 \% \mathrm{Cl}$ \\
\hline Tsimiae & 306 & 21 & 10 & 57 & 357 & 31 & 67 & 3.147 & $0.043^{\star}$ & $\begin{array}{l}0.959- \\
4.806\end{array}$ \\
\hline TCS & 306 & 26 & 5 & 48 & 266 & 31 & 53 & 1.066 & 0.901 & $\begin{array}{l}0.390- \\
2.912\end{array}$ \\
\hline T. godfreyi & 306 & 29 & 2 & 32 & 282 & 31 & 34 & 0.608 & 0.509 & $\begin{array}{l}0.139- \\
2.677\end{array}$ \\
\hline $\begin{array}{l}\text { All trypanosome } \\
\text { species }\end{array}$ & 306 & 9 & 22 & 155 & 159 & 31 & 177 & 4.992 & $0.025^{\star}$ & $\begin{array}{l}0.178- \\
5.012\end{array}$ \\
\hline
\end{tabular}

No = Number of tsetse flies analyzed; TCS = Trypanosoma congolense savannah; $\mathbf{S}+\mathbf{~ = ~ t s e t s e ~ f l i e s ~}$ infected by $S$. glossinidius; $\mathbf{T}+\mathbf{+}$ tsetse flies infected by at least one trypanosome species. $\mathbf{S}+\mathbf{T}+=$ tsetse flies co-infected by $S$. glossinidius and at least one trypanosome species. $\mathbf{S}+\mathrm{T}-\mathrm{=}$ tsetse flies infected by S. glossinidius but without any trypanosome infection; $\mathbf{S}-\mathbf{T}+=$ tsetse flies without $S$. glossinidius but harboring trypanosome infections; S-T- = tsetse flies without trypanosome and S. glossinidius infections. $\mathbf{C l}=$ Confidence Interval; TCS: Trypanosoma congolense savannah; $T$. simiae: Trypanosoma simiae; $T$. godfreyi: Trypanosoma godfrey; $\mathbf{r}=$ coefficient of the generalised linear modeling.

\section{Coinfection of Wolbachia and trypanosomes}


Of the 306 tsetse flies that were considered for tripartite association, $30(9.8 \%)(\mathrm{W}+\mathrm{T}+)$ were coinfected with Wolbachia and at least one trypanosome species. Amongst these 306 tsetse flies, $20(6.6 \%)(\mathrm{W}+\mathrm{T}$-) harbored Wolbachia infections without any trypanosome while 147 (48.0\%) (W-T+) were infected only with trypanosomes. When all trypanosome infections were considered for association studies, no significant association $(r=1.754 ; P=0.185 ; 95 \% \mathrm{Cl}=[0.360-1.219])$ was observed between the presence of Wolbachia and trypanosome infections in G. m. submorsitans (Table 3). Between the presence of Wolbachia and each trypanosome species, no significant association was also observed (Table 3 ).

Table 3

Combined results of Wolbachia and trypanosome infections

\begin{tabular}{|c|c|c|c|c|c|c|c|c|c|c|}
\hline $\begin{array}{l}\text { Trypanosome } \\
\text { species }\end{array}$ & No & $\begin{array}{l}\mathrm{W}+ \\
\mathrm{T}-\end{array}$ & $\begin{array}{l}\mathrm{W}+ \\
\mathrm{T}+\end{array}$ & $\begin{array}{l}\mathrm{W}- \\
\mathrm{T}+\end{array}$ & $\begin{array}{l}\text { W- } \\
T-\end{array}$ & W+ & $T+$ & $\mathbf{r}$ & $\begin{array}{l}\mathrm{P}- \\
\text { value }\end{array}$ & $95 \% \mathrm{Cl}$ \\
\hline T. Simiae & 306 & 39 & 11 & 56 & 239 & 50 & 67 & 1.204 & 0.618 & $\begin{array}{l}0.580- \\
2.497\end{array}$ \\
\hline TCS & 306 & 42 & 8 & 45 & 250 & 50 & 53 & 1.058 & 0.892 & $\begin{array}{l}0.466- \\
2.403\end{array}$ \\
\hline T. godfreyi & 306 & 45 & 5 & 29 & 266 & 50 & 34 & 1.019 & 0.970 & $\begin{array}{l}0.375- \\
2.771\end{array}$ \\
\hline $\begin{array}{l}\text { All trypanosome } \\
\text { species }\end{array}$ & 306 & 20 & 30 & 147 & 148 & 50 & 177 & 1.754 & 0.185 & $\begin{array}{l}0.360- \\
1.219\end{array}$ \\
\hline
\end{tabular}

No = Number of tetse flies analyzed; TCS $=$ Trypanosoma congolense savannah; $\mathbf{W}+\mathbf{+}$ tsetse flies infected by Wolbahia; $\mathbf{T}+=$ tsetse flies infected by at least one trypanosome species. $\mathbf{W}+\mathbf{T}+=$ tsetse flies coinfected by Wolbahia and at least one trypanosome species. $\mathbf{W}+\mathbf{T}-=$ tsetse flies with Wolbahia infection without any trypanosome species; $\mathbf{W}-\mathbf{T}+=$ tsetse flies without Wolbahia but infected by at least trypanosome species; $\mathbf{W}-\mathbf{T}-\mathbf{~}$ tsetse flies without Wolbahia and trypanosome infection; TCS:

Trypanosoma congolense savannah; T. simiae: Trypanosoma simiae; T. godfreyi. Trypanosoma godfreyi; $\mathbf{C l}=$ confidence interval; $\mathbf{r}=$ coefficient of the generalised linear model.

\section{Discussion}

Challenges limiting the appraisal of tsetse microbiome include the difficulties to identify bacterial species in these flies. To fill these gaps in understudied ecological settings, PCR-based method was used to identify S. glossinidius and Wolbachia in wild population of G. m. submorsitans caught in the area of lake Iro in the south of Chad. The identification of S. glossinidius and Wolbachia in wild population of $G$. $m$. submorsitans of Lake Iro is in line with previous studies reporting these two symbiotic microorganisms in wild populations of G. m. morsitans, G. tachinoides, G. p. palpalis, G. pallidipes, G. $f$. quanzensis and G. brevipalpis [2010, 30, 31, 25, 26, 22, 23].

The S. glossinidius infetion rate of $9.0 \%$ obtained in the present study is similar to $9.3 \%$ reported in Liberia for G. p. palpalis [29]. This rate in higher than 1.4\% reported in Zambia for wild populations of $G$. pallidipes [31], but lower than $15.65 \%, 17.5 \%, 54.9 \%$ and $93.7 \%$ previously reported respectively in the 
Democratic Republic of Congo for G. f. quanzensis, in Zambia for G. m. morsitans, in Cameroon for G. $p$. palpalis and in Zambia for $G$. brevipalpis [7, 31, 23]. These results confirm the high heterogeneity of $S$. glossinidius infection rates according to tsetse species [31, 7]. Nevertheless, reliable comparisons between data from by different studies require to understand the study designs. In the present study, $S$. glossinidius was identified in tsetse body while in other studies, whole tsetse or parts of the insect such as the abdomen, the thorax and the legs were used. The heterogeneity observed in the S. glossinidius infection rates could be explained by some variations in methodological approaches, the intrinsic characters of each tsetse species and environmental factors (vegetation, humidity, temperature) encountered in different ecological settings. In natural conditions where environmental factors vary and have impacts on the biology of tsetse flies, the relationship between tsetse and its symbiotic microorganisms is affected. As the survival and the transmission of these symbiotic micro-organisms are linked to tsetse biology because of their limited metabolic capacities, each environmental factor affecting the biology of tsetse could change its interactions with symbiotic micro-organisms. In such a scenario, $S$. glossinidius could not undergo horizontal transmission with the same efficiency and in consequence, its infection rates could vary with environmental factors. When environmental variations are removed like in experimental studies or in insectarium [33], the symbiotic association between tsetse and its symbionts is not affected. As already reported in G. p. gambiense and G. m. morsitans, high vertical transmission and high infection rates of symbiotic micro-organisms are observed in tsetse flies [11].

The significant association $(r=4.992 ; P=0.025 ; 95 \% \mathrm{Cl}=[0.178-5.012])$ observed between S. glossinidius and trypanosome infections indicates that the presence of $S$. glossinidius seems to favor trypanosome infections in G. m. submorsitans of the area of lake Iro in the south of Chad. Although these results are not in agreement with those reporting no significant association between the presence of $S$. glossinidius and trypanosome infections in G. austeni [20], G. brevipalpis, G. m. morsitans and G. pallidipes [31], our findings are in line with those reported in G. p. palpalis of some sleeping sickness foci of Cameroon [7] and other tsetse species $[20,8,11]$. The discrepancies observed in the tripartite association between tsetse, $S$. glossinidius and trypanosomes may result from differences in the biology of different tsetse species as well as the bioclimatic conditions impacting the relationship between tsetse and its symbiotic micro-organisms. Moreover, our results showing significant association $(r=3.147 ; P=0.043 ; 95 \% \mathrm{Cl}=$ [0.178-5.012]) between S. glossinidius and T. simiae, but no association for other trypanosome species identified in this study suggest that the tripartite association between tsetse, $S$. glossinidius and trypanosomes could vary according to trypanosome species. Better understanding these tripartite associations requires more in-depth investigations on wild populations of different tsetse species of various tsetse infested areas.

The identification of Wolbachia in wild populations of G. m. submorsitans may have some implications in the development of new vector control strategies. On the basis of its capacity of inducing cytoplasmic incompatibility and to be transmitted from mother to offspring, Wolbachia can be genetically modified with the objective of producing biomolecules able to interfer with the establishment and/or the development of trypanosomes in tsetse flies. If that occurs, the vectorial competence of tsetse will be 
affected and disease transmission could be blocked through genetically modified Wolbachia strains that conferred resistance to tsetse fly [26].

The overall Wolbachia infection rate of $14.5 \%$ obtained in the present study is lower than $25.32 \%, 44.3 \%$, $88.8 \%, 98 \%$ and $100 \%$ reported respectively in G. p. palpalis [26], G. f. fuscipes [27], G. f. Quanzensis[23], G. austeni $[33,20]$ and G. m. morsitans [34]. These results show a certain heterogeneity in the Wolbachia infection rates according to tsetse species. As already reported by Kante et al. [26], this heterogeneity could be related to specific biological characteristics of each tsetse subspecies. For identical stimulus, it has been reported that interactions between tsetse fly and its symbiotic micro-organisms vary according to specific biological response of each tsetse species or subspecies [26]. Such variations affect not only the interactions between tsetse and its symbiotic micro-organism, but also the Wolbachia infection rates. Some discrepancies observed in the Wolbachia infection rates could be explained by some differences in the study design as well as the analytical methods. In the present study, Wolbachia was searched in tsetse body (whole tsetse without legs, wings and proboscis) while in other studies, investigations were undertaken on isolated tissues or whole tsetse fly. In addition, the fact that one molecular marker was used to detect Wolbachia infections has probabily underestimated its infections rates. Indeed, in tsetse flies from the same ecological setting, Kante et al. [26] reported significant differences in the Wolbachia infection rates when different molecular markers were used. In Camerooun for instance, the detection of wsp gene was two-fold more sensitive in tsetse from Campo while 16S rDNA showed higher sensitivity in flies from Fontem [26]. In addition to differences in the sensitivity of molecular markers, the technical approaches could also have impacts on the Wolbachia infection rates. If the density of Wolbachia in some G. m. submorsitans is below the detection threshold of standard PCR-based method, some infections could pass undetected. Wamwiri et al. [20] highlight a high density of Wolbachia in G. austeni populations from Kenya and a low density in the same tsetse species of South Africa. In addition, a low density of Wolbachia has been reported in Rhagoletis cerasi [35] and Drosophila paulistorum [36]. While searching for sensitive and reliable markers or tools for Wolbachia identification remains a goal to achieve, the use of one marker or standard PCR-based method may lead to an underestimation of the real Wolbachia infection rates.

The $9.8 \%$ of G. m. submorsitans harboring co-infections of Wolbachia and trypanosomes is low compared to $29.84 \%$ and $26 \%$ reported respectively in G. p. palpalis [26] and G. tachinoides in Cameroun [22]. Although the technical approach and the study design could partially explain this low co-infection rate, such co-infections are probably not common in G. m. submorsitans of lake Iro. The absence of significant association $(r=1.754 ; P=0.185 ; 95 \% \mathrm{Cl}=[0.360-1.219])$ between Wolbachia and trypanosome infections suggests that the presence of this bacterium does not seem to be an obstacle for the establishment of trypanosomes. These results are in agreement with those of Kante et al. [26] reporting no association in G. p. palpalis from in sleeping sickness foci of Cameroon. They contrast data of Alam et al. [27] showing a negative correlation between Wolbachia and trypanosome infections and suggesting that the presence of this bacterium prevent trypanosome infections in $G$. $f$. fuscipes. The tripartite association between tsetse, Wolbachia and trypanosomes seems to vary according to tsetse species or subspecies. 
Results of the present study showing that only $2.31 \%$ of tsetse flies were co-infected by Wolbachia and $S$. glossinidius are in agreement with the $5.43 \%$ previously reported in G. f. quanzensis [23]. They indicate that co-infections between Wolbachia and S. glossinidius are rare in wild populations of G. $\mathrm{m}$. submorsitans. The co-infection rate between $S$. glossinidius and Wolbachia is probably underestimated in the present study because the molecular markers used have been reported to be of low sensitiviety, especially when only one marker was used to detect symbiotic microorganisms. The low co-infection rate revealed between S. glossinidius and Wolbachia can be also explained by the biological effects of each of these bacteria. Indeed, association studies revealed that the presence of $S$. glossinidius seems to favor trypanosome infections while no association was reported between Wlobachia and trypanosome infections. In other studies, the negative correlation reported between trypanosomes and Wolbachia infections suggested that the presence of Wolbachia seems to prevent trypanosome infections [27, 30]. These observations suggest that some antagonistic actions, resulting from different biological actions of Wolbachia and S. glossinidius could occur in tsetse fly during trypanosome infections.

Our investigations on tripartite associations were based on presence/absence of trypanosome or $S$. glossinidius or Wolbachia. Instead of focusing on this presence/absence, the genetic characterization of S. glossinidius or Wolbachia strains could provide additional values to decript these associations. In previous investigations, it has been reported that the tripartite association could be affected by specific genotypes of $S$. glossinidius and some trypanosome species such as T. b. gambiense and T. b. brucei [8]. For some trypanosome species, specific $S$. glossinidius genotypes have been reported to affect the vectorial competence of G. p. gambiensis and G. m. morsitans [11]. Genetic characterization of bacteria populations could provide additionnal data to improve knowledge on this tripartite association, and also to better understand the real contribution of symbiotic microorganisms (S. glossinidius or Wolbachia) in the vectorial competence of tsetse flies. To obtain the real overview of the vector competence of tsetse flies, it is also important to take into consideration other factors such as the level of lectin in the tsetse gut at the time of parasite uptake, the fly species, the age, the teneral status of tsetse and its first blood meal on a non-infected host because these factors affect its ability to be infected and could mitigate the influence of symbiotic micro-organisms. Such factors could play a significant role in the success or failure of parasite establishment because the processes leading to this establishment involve complex interactions between these factors [13].

\section{Conclusion}

This study revealed S. glossinidius and Wolbachia in wild population of G. m. submorsitans of Lake Iro in the south of Chad. It showed that few tsetse flies harbor co-infections of Wolbachia and S. glossinidius. Co-infections of Wolbachia and trypanosomes or S. glossinidius and trypanosomes are not common in G. m. submorsitans. No association was revealed between Wolbachia and trypanosomes while significant association was observed between the presence of $S$. glossinidius and trypanosome infections. Decrypting the tripartite association involving tsetse, symbionts and trypanosomes requires additional studies aiming to understand the relationship between haplotypes or genotypes of Wolbachia and/or S. glossiniduis and trypanosome infections. 


\section{Methodology \\ Study area}

Tsetse flies were caught in the area of Lake Iro, along the Salamat River in the Middle Chari region of the south of Chad (Fig. 1). This area is considered as a buffer zone of the Zakouma national park where domestic and wild animals can meet. It is located between latitude $09^{\circ} 59^{\prime} \mathrm{N}$ and longitude $019^{\circ} 26^{\prime} \mathrm{E}$ and has a climate of Sudano-Sahelian type with one dry season (November to April) and one rainy season (May to October). This locality has an average annual temperature and relative humidity of respectively $27^{\circ} \mathrm{C}$ and $50 \%$ [37]. The rainfall varies from $800 \mathrm{~mm}$ to $1200 \mathrm{~mm}$ per year [38] and the vegetation is dominated by floodplains and dense forests containing shrubs. The hydrographic network is mainly dominated by the Lake Iro and the Salamat River, which flows into the Chari River that feeds Lake Chad in the north. The population is estimated at 174,195 inhabitants who are mainly herdermen, farmers and fishermen [39].

\section{Entomological survey}

Three entomological surveys were performed in November 2018, February 2019 and February 2020. During these surveys, biconical traps [40] were set up along the Salamat River, especially where the bioclimatic conditions were considered favourable for the development of tsetse flies. The geographical coordinates of each tsetse trap were recorded using a global positioning system (GPSMAP® 60CSX Garmin). The temperature and the relative humidity were recorded using a thermohygrometer (EasyLog TH, Lascar, Whiteparish, UK). Tsetse flies were collected each day at $9 \mathrm{am}$. The collected flies were morphologically identified and their sex and species determined.

\section{Collection of tsetse fly organs}

From each tsetse fly, the wing pairs (for morphometric analyses) as well as the legs (for genetic studies) were removed and introduced separately into dry microtubes. Thereafter, the proboscis (for the identification of trypanosome species) and the remaining body (for the identification of trypanosome species and symbiotic micro-organisms) of each tsetse were collected and each of them placed in a $1.5 \mathrm{~mL}$ cryotube containing $200 \mu \mathrm{l}$ of nucleic acid preservative solution $(25 \mathrm{mM}$ sodium citrate, $10 \mathrm{mM}$ EDTA and 70\% ammonium sulphate). The dissecting tweezers were decontaminated in $5 \%$ solution of sodium chloride and then rinsed with distilled water after dissection of each tsetse fly. In the field, the samples were preserved at 4 degrees and once in the Laboratory, they were stored at -80 degree.

\section{DNA extraction from the tsetse body}

DNA was extracted from the body of each tsetse fly using $5 \%$ chelex-resin (Chelex 100, Bio-rad). Briefly, each tsetse body was removed and put into a new $1.5 \mathrm{~mL}$ microtube. This tsetse body was crushed using the tip of the Pasteur pipette. Thereafter, $100 \mu$ of chelex $5 \%$ solution were added. Each microtube was vortexed and incubated at $56^{\circ} \mathrm{C}$ for 30 minutes in a Thermomixer. After this incubation, the microtubes were vortexed and reincubated at $95^{\circ} \mathrm{C}$ for 5 minutes. These microtubes were subsequently centrifuged at 
10,000 rpm for 1 minute. The concentration of DNA extracts was determined using a Nanodrop 1000 (Thermo Scientific-Germany). DNA extracts were stored at $-20^{\circ} \mathrm{C}$ for molecular analysis.

\section{Tsetse species identification}

The confirmation of each tsetse fly was performed by amplifying and sequencing the Cytochrome oxidase I (COI) gene. This amplification was done using C01-sense (5' TTG ATTTTT TGG TCA TCC AGA AGT-3') and C01-non-sense (5'-TGA AGC TTA AAT TCA TTG CAC TAA TC-3') premers designed by Dyer et al. [41]. Briefly, PCR reaction was performed in a final volume of $25 \mu \mathrm{L}$ containing $2.5 \mathrm{U}$ of dream taq polymerase, $2.5 \mu \mathrm{L}$ of the dream taq buffer (10X), $0.2 \mathrm{mM}$ of dNTPs (all provided by Thermo Scientific, Dreieich, Germany), $2 \mu \mathrm{M}$ of each primer and $1 \mu \mathrm{L}$ of DNA extract. The amplification program consisted of an initial denaturation step of $95^{\circ} \mathrm{C}$ for $5 \mathrm{~min}$, followed by 35 cycles. Each of these cycles was made up of a denaturation step at $94^{\circ} \mathrm{C}$ for $1 \mathrm{~min}$, an annealing step at $55^{\circ} \mathrm{C}$ for $1 \mathrm{~min}$ and an elongation step at $72^{\circ} \mathrm{C}$ for $1 \mathrm{~min}$. This was followed by a final elongation at $72^{\circ} \mathrm{C}$ for $5 \mathrm{~min}$.

After each PCR reaction, $20 \mu \mathrm{L}$ of PCR products were checked by electrophoresis on $1.5 \%$ agarose gel containing $3 \mu \mathrm{L}$ G-stain (Serva, Heidelberg, Germany). The agarose gel was stained, visualized under ultraviolet light (UV) and photographed.

Each sample for which a DNA fragment of $930 \mathrm{bp}$ was revealed by electrophoresis was selected and the remaining amplicons purified using GeneJet DNA purification kit (Thermo Scientific, Dreieich, Germany). This purification was performed following the manufacturer's instruction. Each purified COI DNA fragment was sequenced by a commercial company (SeqLab, Göttin- gen, Germany). To identify each tsetse species or subspecies, each sequenced fragment of $\mathrm{COI}$ was compared to those available in the database (Genbank) of the National Center for Biotechnology Information (NCBI). This was done through a BLAST search.

\section{Identification of S. glossinidius.}

Sodalis glossinidius was identified by PCR using the pSG2 primers (pSG2-sense: 5'-

TGAAGTTGGGAATGTCG-3': pSG2-non-sense: 5'-AGTTGTAGCACAGCGTGTA-3') as previously described by Darby et al. [42]. The PCR reaction was performed in a final volume of $15 \mu \mathrm{L}$ containing $1.5 \mu \mathrm{L}$ of Dream taq buffer, $0.5 \mu \mathrm{L}$ of Dream taq polymerase ( $5 \mathrm{U} / \mu \mathrm{L}), 0.3 \mu \mathrm{L}$ of dNTPs $(10 \mathrm{mM} / \mu \mathrm{L}), 0.5 \mu \mathrm{L}$ of each primer $(100 \mathrm{mg} / \mu \mathrm{L})$ and $0.5 \mu \mathrm{L}$ of DNA extract. The amplification program consisted of an initial denaturation step at $94^{\circ} \mathrm{C}$ for 3 minutes followed by 30 cycles. Each of these cycles was made up of a denaturation step at $94^{\circ} \mathrm{C}$ for $30 \mathrm{sec}$, an annealing step at $51^{\circ} \mathrm{C}$ for $45 \mathrm{sec}$ and an elongation step at $72^{\circ} \mathrm{C}$ for $1 \mathrm{~min}$. The final elongation was done at $72^{\circ} \mathrm{C}$ for $5 \mathrm{~min}$. For each PCR reaction, positive and negative controls were used. In the positive control, purified genomic DNA of $S$. glossinidius was used while in the negative control, the DNA solution was replaced by nuclease free water.

After PCR reactions, amplicons were separated by electrophoresis on $2 \%$ agarose gel containing $3 \mu \mathrm{L}$ of G-stain (Serva, Heidelberg, Germany). The electrophoresis was performed at 100 volt for $60 \mathrm{~min}$. At the 
end of each electrophoresis, the gel was visualized under UV light. Samples were considered positive for S. glossinidius if a DNA fragment of 120 bp was observed.

The presence of $S$. glossinidius was confirmed by sequencing 5 positive randomly selected samples. For these 5 selected samples, the same PCR was performed in a final volume of $50 \mu \mathrm{L}$. After electrophoretic separation of PCR products of these 5 samples, amplicons were purified from agarose gel using the GenJet purification kit following the manufacturer's instructions. The purified PCR products were cloned into pJET 1.2 cloning vector (Thermo Scientific) following the manufacturer's instructions. Recombinant clones or clones containing PSG2 sequences were identified by PCR using PSG2 primers. PCR reactions were carried out in the same conditions as described above.

Recombinant clones were picked up from petri dish and cultured overnight at $37^{\circ} \mathrm{C}$ into LB medium supplemented with ampicillin $(100 \mu \mathrm{g} / \mathrm{ml})$. The bacteria cultures were centrifuged at $4500 \mathrm{xg}$ for $15 \mathrm{~min}$ at $4^{\circ} \mathrm{C}$ and the pellet was recorvered. Plasmid DNA was purified from each pellet using the GeneJET Plasmid MiniPrep Kit (Thermo Fischer Scientific). The Plasmidic DNA was send for sequencing that was performed by a commercial company (institute SeqLab, Göttingen, Germany).

\section{Molecular identification of Wolbachia}

Wolbachia was identified by amplifying the wsp (Wolbachia surface protein) gene as described by Baldo et al. [43]. The amplification was done using wsp-sense (5'-GTCCAATARSTGATGARGAAAC-3') and wspnon-sense (CYGCACCAAYAGYRCTRTAAA-3') primers designed by Baldo et al. [43]. PCR reaction was performed in a total volume of $15 \mu \mathrm{L}$ containing $1.5 \mu \mathrm{L}$ of Dream taq buffer, $0.1 \mu \mathrm{L}$ of Dream taq polymerase $(5 \mathrm{U} / \mu \mathrm{L}), 0.3 \mu \mathrm{L}$ of dNTPs $(10 \mathrm{mM} / \mu \mathrm{L}$ ) (all from Thermo Fischer Scientific) and $0.5 \mu \mathrm{L}$ of each primer $(100 \mathrm{mg} / \mu \mathrm{L})$ (provided by Sigma-Aldrich, Darmstadt, Germany) and $0.5 \mu \mathrm{L}$ of DNA extract. The amplification program consisted of an initial denaturation step for 5 minutes at $95^{\circ} \mathrm{C}$ followed by 35 cycles. Each cycle was made up of a denaturation step at $94^{\circ} \mathrm{C}$ for $30 \mathrm{sec}$, an annealing step at $53^{\circ} \mathrm{C}$ for $30 \mathrm{sec}$ and an elongation step at $72^{\circ} \mathrm{C}$. The final elongation was done at $72^{\circ} \mathrm{C}$ for 10 minutes. During each PCR, positive and negative controls were used. The positive control was a purified genomic DNA of Wolbachia while in the negative control, the DNA solution was replaced by nuclease free water.

Amplicons of each PCR reaction were subjected to an electrophoresis that was carried out as described above. Samples were considered as having Wolbachia infections if a DNA fragment of $513 \mathrm{bp}$ was identified after electrophoresis. As for $S$. glossinidius, the presence of Wolbachia was confirmed by sequencing amplicons of 5 positive randomly selected samples. For each of these samples, another PCR was performed in a volume of $50 \mu \mathrm{L}$. The amplicons were purified by the GenJET purification kit (Thermo Fischer Scientific). The purified PCR product was directly sent to a commercial company (SeqLab, Göttingen, Germany) for sequencing.

Geneious Pro version 5.5.9 software was used to store, organize and analyze the sequences obtained from PSG2 and wsp genes of S. glossinidius and Wolbachia, respectively. The presence of S. glossinidius 
and Wolbachia was confirmed by BLAST searching respectively of $\mathrm{pSG} 2$ and wsp sequences at the data base (Genbank) of the National Center for Biotechnology Information (NCBI). After BLAST search, a sequence was considered as belonging to $S$. glossinidius if the sequenced DNA fragment of 120 bp had at least $98 \%$ of similarity with those of $S$. glossinidius available in the data base. For Wolbachia, the nucleotide sequence of the sequenced DNA fragment of $513 \mathrm{bp}$ must also have at least $98 \%$ of similarity with the sequence of Wolbachia available in the database.

\section{Statistical analysis}

The R software was used for statistical analysis [44]. The Chi-square test was used to compare the infection rates of $S$. glossinidius and Wolbachia according to sex and sampling periods. The test was considered significant when the P value was below 0.05 . Generalised linear modelling ( $\mathrm{glm}$ ) with $95 \%$ confidence intervals (Cls) was used to evaluate the association between symbiotic microorganisms and trypanosome infections. For association studies, data on trypanosome infections were retrieved for results of Djoukzoumka et al. (submitted). To carry out these association studies, T. vivax was excluded because its lifecycle is restricted to the mouthparts of tsetse flies.

\section{Abbreviations}

ITS-1: Inner Transcribed Spacer 1; pSG 2: Plasmid Sodalis glossinidius 2, wsp: Wolbachia surface protein; AAT: Animal African Trypanosomiasis; HAT: Human African Trypanosomiasis.

\section{Declarations}

\section{Acknowledgements}

The authors thank sincerely the "Deutsche Forschungsgemeinschaft (DFG)" for the financial support of this project. We thank also staffs of the Laboratory for Biomolecular Interaction of the University of Bremen in Germany for their technical assistance and fruitful practical discussions during the laboratory work. We also thank the regional representation of the Ministry of Livestock of Moyen Chari in Chad, the military and traditional authorities for their guidance and support during sample collection, the University of Dschang through the Molecular Parasitology and Entomology subunit of the Department of Biochemistry.

\section{Funding}

This study was financed by "Deutsche Forschungsgemeinschaft (DFG Ke428/13-1)" through the Project entitled "Animal African Trypanosomiasis in Humans, An emergency zoonosis?"

\section{Author's contributions}

GS, DS, VKP, SK and HMH contributed to the design of the Project. DS, IMAM, SK, PB and YMM contributed in sample collection and laboratory analysis. DS, STK and GS analyzed the data. DS and GS 
and wrote the Manuscript.

\section{Availability of data and materials}

All data generated and/or analyzed during this study are included in this article.

\section{Ethics approval and consent to participate}

Not applicable.

\section{Consent for publication}

Not applicable.

\section{Competing Interests}

The authors declare that they have no competing of interests

\section{References}

1. Büscher P, Cecchi G, Jamonneau V, Priotto G. Human African Trypanosomiasis. Lancet. 2017; 390: 2397-4091.

2. WHO. 2020. Sustained declined in sleeping sickness cases puts elimination within reach. World Health Organization: Geneva, Switzerland. https://www.who.int/news/item/23-06-2020-sustaineddecline-in-sleeping-sickness-cases-puts-elimination-within-reach

3. WHO. Accelerating work to overcome the global impact of neglected tropical diseases: a roadmap for implementation. World Health Organization: Geneva, Switzerland 2012; 42p.

4. Holmes P. Tsetse-transmitted trypanosomes - Their biology, disease impact and control. J Invertebr Pathol.2013; 112:S11-4.

5. Schofield CJ and Kabayo JP. Trypanosomiasis vector control in Africa and Latin America. Parasite and Vector. 2008; 1(21).

6. Allsopp R. Options for vector control aigainst trypanosomiasis in Africa. Trends in Parasitology. 2001; 17(1): 15-19

7. Farikou O, Njiokou F, Mbida Mbida JA, Njitchouang GR, Djeunga HN, Asonganyi T, Simarro P, Cuny G, Geiger A. Tripartite interactions between tsetse flies, Sodalis glossinidius and trypanosomes. An epidemiological approach in two historical human African trypanosomiasis foci in Cameroon. Infection, Genetics and Evolution. 2010; 10, 115-121.

8. Geiger A, Ravel S, Mateille T, Janelle J, Patrel D, Cuny G, Frutos R. Vector competence of Glossina palpalis gambiensis for trypanosome brucei s.l and genetic diversity of the symbiont Sodalis glossinidius. Molecular Biology and Evolution. 2007; 24: 102-109 
9. Wang J. Wu Y and Aksoy S. Interaction between mutualist Wiggleworthia and tsetse peptidoglycan recognition protein (PGRP-LB) influence trypanosome transmission, Proc Natl Acad Sci USA. 2009; 106 (29): 12133-12138.

10. Abd-Alla AMM, Paker AG, Vreysen MJB, Bergoin M. Tsetse salivary gland hypertrophy virus.hope or hindrance fort setse control? PLoS Negl Trop Dis. 2011; 5(8).

11. Geiger A, Ravel S, Frutos R, and Cuny G. Sodalis glossinidius (Enterobacteriaceae) and vectorial competence of Glossina morsitans morsitans for Trypanosoma congolense Savannah type. Current Mycrobiology. 2005; 51 :35-40

12. Pais R, LohsC, Wu Y, Wang $\mathrm{J}$ ans Aksoy S. The obligate mutualist Wiggleworchia glossinidia influences reproduction, digestion and immunity process in its host, the tsetse fly. Applied en Enviromental Microbiology. 2008; 74: 5965-5974.

13. Welburn SC, Maudlin I (1993). The nature of the teneral state in Glossina and its role in the acquisition of trypanosome infection in tsetse. 1993; 86(5): 529-36P.

14. Hamidou Soumara I, Tchikaya B, Loriod B, Richet P, Geiger A. Identification of overexpressed genes in Sodalis glossinidius inhabiting trypanosome-infected self-cured tsetse flies. Frontiers in Microbiology. 2014; 5(255):1-8.

15. Dale C, Welburn SC. The endosymbionts of tsetse flies: manipulating host-parasite interactions. International Journal for Parasitology; 2001 31: 628-631.

16. Ross PA, Wiwatanaratanabutr I, Oxfort JK, White VL, Endersby-Harshman NM, Hoffmann AA. Wolbachia infection in Aedes aegypti differ markedly int their response to cyclical heat stress. PLoS pathogens. 2017; 13(1); e1006006. https://doi.org/10.1371/journal.ppat.1006006

17. Bandi C, Anderson TJ, genchi C, Blaxter. Phylogeny of Wolbachia in filarian nematodes. Proc. R. Soc. Lond. B. 1998; 265: 2407-2413.

18. Kelly M, Shauna L, Ramalho MO, Moreau CS. Diversity of Wolbachia associetd with the giant turtle ant, Cephalote atratus. Current Microbiology. 2019; 76, 1330-1337.

19. Stouthamer RJA, Breeuwer GD. « Wolbachia pipientis: microbial manipulator of arthropod reproduction », Rev. Microbiol. 1999; (53) 71-102

20. Wamwiri FN, Usma A, Thande PC, Aksoy E, Ngure RM, Aksoy S, Puma JO \& Mirilla GA. Wolbachia, Sodalis and trypanosome co-infections in natural populations of Glossina austeni and Glossina pallidipes. Parasite and Vector. 2013;6(236)

21. O'Neill S L, Gooding R H, Aksoy S (1993). Phylogenetically distant symbiotic microorganisms reside in Glossina midgut and ovary tissues. Med Vet Entomol. 1993; 7:377-383.

22. Kame-Ngasse GI, Njiokou, Melachio-Tanekou TT, Farikou O, Simo G, and Geiger A. Prevalence of symbionts and trypanosome infections in tsetse flies of two villages of the "Faro and Déo" division of the Adamawa region of Cameroon.BMC Microbiology. 2018 ; 18: 159.

23. Simo G, Kanté ST, Madinga J, Kame G, Farikou O, llombe G, Geiger A, Lutumba P \& Njiokou F. Molecular identification of Wolbachia and Sodalis glossinidius in the midgut of Glossina fuscipes quanzensis from the Democratic Republic of Congo. 2019; $26,5$. 
24. Aksoy E, Telleria EL, Echodu R, Wu Y, Okedi LM, Weiss BL, Aksoy S, Caccone A. Analysis of multiple tsetse fly population in Uganda reveals limited diversity ans species specifique gut microbiota. Applied and Environnemental microbiology. 2014; 80, 4301-4312.

25. Kante TS, Farikou O, Njiokou F, and Simo G. Prevalence of Sodalis glossinidius and different trypanosome species in Glossina palpalis palpalis caught in the Fontem sleeping sickness focus of the southern Cameroon. Parasite. 2018a; 25, 44

26. Kante TS, Melachio T, Ofen Elvis, Njiokou F, and Gustave Simo. Detection of Wolbachia and different trypanosome species in Glossina palpalis palpalis populations from three sleeping sickness focus in Southern Cameroon. Parasite and Vector. 2018b; 11:6-30.

27. Alam U, Medlock J, Brelsfoard C, Pais R, Lohs C, Balmand S, Carnogursky J, Heddi A, Takac P, Galvani A, Aksoy S. Wolbachia symbiont infections induce strong cytoplasmic incompatibility in the Tsetse Fly Glossina morsitans. PLoS Pathogens. 2011; 7(12), e1002415.

28. Odeniran PO, Macleod ET, Ademola MI, Welburn SC. Endosymbionts interaction with trypanosomes in Palpalis group of Glossina captured in southwest Nigeria. Parasitology Internaltionale. 2019 ; 70(1) :64-69.

29. Maudlin I, Welburn SC, Mehlitz D. The relationship between rickettsia-like-organisms and trypanosome infections in natural populations of tsetse in Liberia. Tropical Medicine and Parasitology. $1990 ; 41,265-267$.

30. Aksoy S, Rio RV. Interactions among multiple genomes: tsetse, its symbionts and trypanosomes. Insect Biochemistry and Molecular Biology. 2005; 35(7):691-698.

31. Dennis JW, Durkin SM, Horsley DJE, Hamill LC, Anderson NE, MacLeod ET. Sodalis glossinidius prevalence and trypanosome presence in tsetse from Luambe National Park, Zambia. Parasites and Vector. 2014; 7, 378.

32. Rio RVM, Yi-neng W, Filardo $G$ and Aksoy S. Dynamics of multiple symbiot density regulation during host development: tsetse and its microbial flora. Proceeding of the Ryal Society of London Series B: Biological Sciences. 2006; 273 :805-814

33. Cheng Q, Aksoy S. Tissue tropism, transmission and expression of foreign genes in vivo in midgut symbionts of tsetse flies. Insect Mol Biol. 2000; 8:125-132.

34. Doudoumis V, Tsiamis G, Wambiri F, Brelsfoard C, Alam U, Aksoy E, Delapera S, Abd-Alla A, Ouma J, Takac p, Aksoy S, Bourtzis. Detection and charaterization infection in laboratiry and natural populations of different species of tsetse flies (Genus Glossina). BMC Microbiology. 2012; 12 :1-13

35. Bakovic V, Schebeck M, Telschow A, Stauffer C, Shuler H. Spacial spread of Wolbachia in Rhagoletis cerasi Bilogy letters. 2018; 14: 2018016. http://dx.doi.org/10.1098/rsbl.2018.0161Aksoy S, Caccone A, Galvani AP, Okedi LM. Glossina fuscipes populations provide insights for Human African Trypanosomiasis transmission in Uganda. Trends in Parasitology. 2013; 29, 394-406.

36. Artohofer W, Riegler M, Schneider G, Krammer M, Miller WJ, Stauffer C. Hidden Wolbachia diversity in field populations of the European cherry fruit fly, Rhagoletis cerasi (Diptera, Tephritidae). Molecular Ecology. 2009; $18: 3816-30$ 
37. Boyer JD, Rouche CN, Cres A, Servat E, Paturel J, and Mahé G. SIEREM an environnmental information systemfor water resources, 5th World FRIEND Conference. La Havana. Cuba. 2006; 1925.

38. Paigbc. Pla d'aménagement intégré à base communautaire, site de Boum Kebir. Ministère de l'Environnement et des Ressources halieutiques, direction des pêches et de l'aquaculture : 2012 ; $110 \mathrm{p}$

39. Raimond C, Florence Sylvestre, Zakinet D et Abderamane M. Tchad des lacs: les zones humides sahéliennes au défi du changement global. IRD Éditions. 2019. DOI : 10.4000 irdeditions.30450.

40. Chalier Laveissiere. Amélioration du rendement du piège biconique pour glossines (Diptera, Glossinidae) par l'emploi d'un cône inférieur bleu. Entomologie médicale et Parasitologie. 1973; 3 : 283-286.

41. Dyer NA, Lawton S.P, Ravel S, Choi KS, Lehane M.J, Robinson A.S, Okedi LM, Hall MJ, Solano P, Donnelly MJ. Molecular phylogenetics of tsetse flies (Diptera: Glossinidae) based on mitochondrial (COI, 16S, ND2) and nuclear ribosomal DNA sequences, with an emphasis on the palpalis Mol. Phylogenet. Evol. 2008; 49: 227-239.

42. Darby AC, Lagnel J, Matthew CZ, Bourtzis K, Maudlin I, Welburn SC. Extrachromosomal DNA of the Symbiont Sodalis glossinidius, Journal of Bacteriology. 2005; 14(187).

43. Baldo L, Hotopp JCD, Jolley KA, Bordeistein SR, Biber Sa, Choudhuri RR, Hayashi C, Maiden MCJ, Tettelin $\mathrm{H}$, Werren $\mathrm{JH}$. Multilocus sequence typing system for the endosymbiont Wolbachia pipientis. Applied and Environnemental Microbiology. $2006 ; 72: 155-165$

44. R Core Team. R: A language and environment for statistical computing. Vienna, Austria: $R$ Foundation for Statistical Computing. ISBN 3-900051-07-0. 2010. Retrieved from http://www.Rproject.org/

\section{Figures}




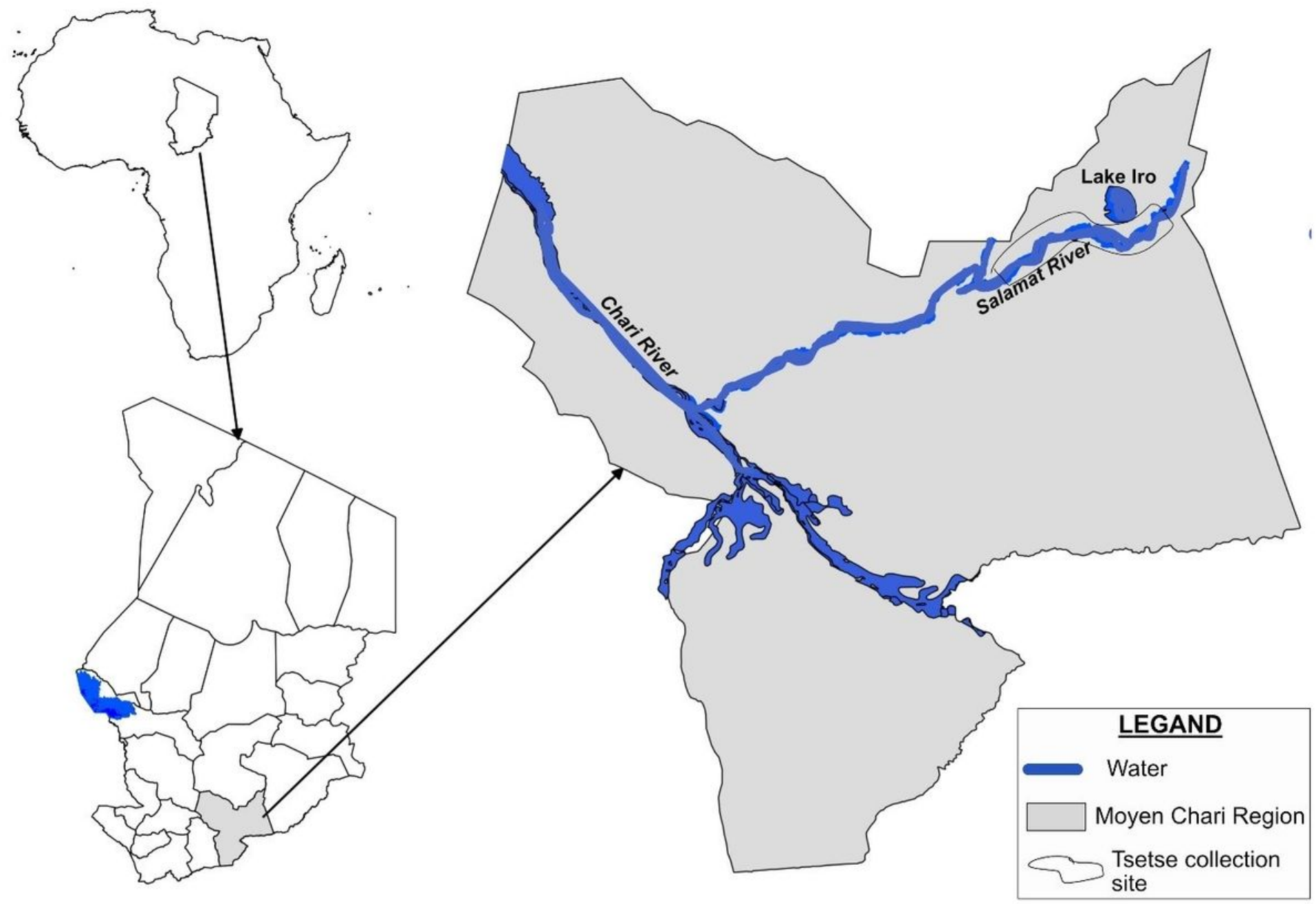

Figure 1

Tsetse flies were caught in the area of Lake Iro, along the Salamat River in the Middle Chari region of the south of Chad. Note: The designations employed and the presentation of the material on this map do not imply the expression of any opinion whatsoever on the part of Research Square concerning the legal status of any country, territory, city or area or of its authorities, or concerning the delimitation of its frontiers or boundaries. This map has been provided by the authors.

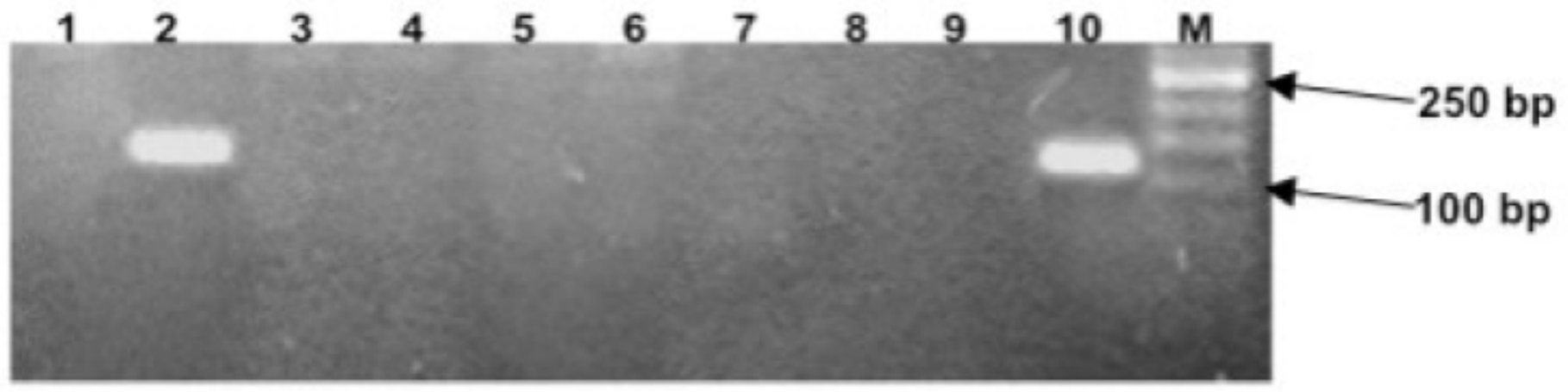




\section{Figure 2}

Electrophoretic profile illustrating the separation of amplified products of PSG2 gene of S. glossinidius. 1, 3, 4, 5, 6, 7 and 8: samples without S. glossinidius infection; 2: sample with S. glossinidius infection; 9: negative control; 10: positif control (genomic DNA of S. gossinidius); M: GeneRuler 50 bp Ladder (Thermo Fischer Scientific).

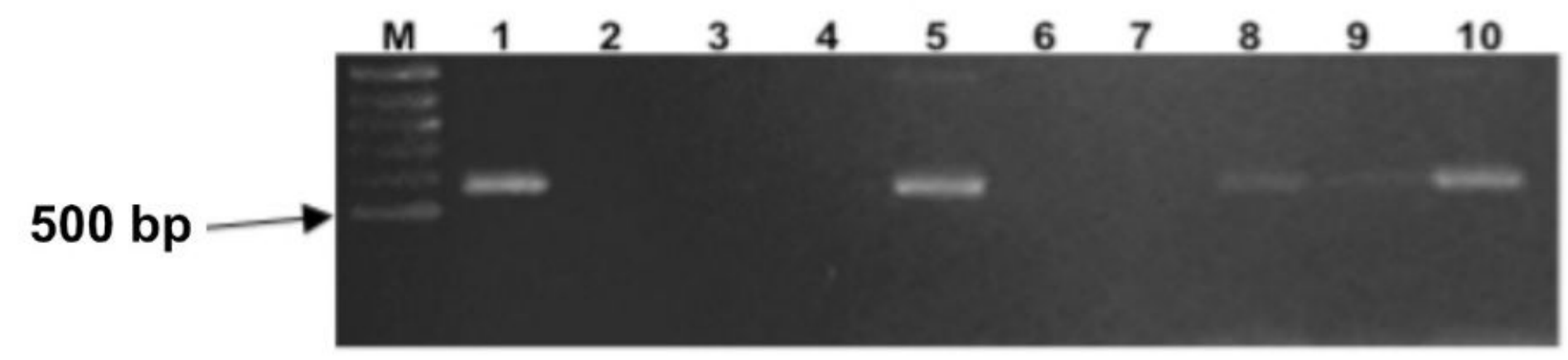

\section{Figure 3}

Electrophoretic profile showing the amplified DNA fragment of WSP gene of Wolbachia. M: Massruler DNA ladder mix (thermo Fischer Scientific); 1: positif control (genomic DNA of Wolbachia sp.); 2: negative control; 5, 8, 9 and 10: samples with Wolbachia infections; 3, 4, 6 and 7: samples without infection due to Wolbachia. 